\title{
LHCb Upgrade - Physics Case and High Luminosity Detector Optimisation
}

\author{
Paula Collins, On behalf of the LHCb collaboration* \\ CERN \\ E-mail: Paula.Collins@cern.ch
}

LHCb, the dedicated B physics experiment at the LHC, plans to increase its data taking capabilities by a factor of up to 20 , by running at a higher luminosity with an upgraded detector around 2016. The envisaged maximum luminosity of $1-2 \times 10^{33} \mathrm{~cm}^{-2} \mathrm{~s}^{-1}$ is compatible with both LHC and SLHC running. The LHCb front end electronics will be upgraded to allow a full readout of the detector at $40 \mathrm{MHz}$. Upgrades and replacements are necessary for the silicon detectors, and for the photo multiplier read out of the RICH detectors. This article presents the physics case for the luminosity increase and detector upgrades a description of the proposed detector modifications.

12th International Conference on B-Physics at Hadron Machines

September 7-11, 2009

Heidelberg, Germany.

* Speaker. 


\section{Introduction to the LHCb Upgrade}

$\mathrm{LHCb}[1]$ is an experiment installed at one of the four collision points of the Large Hadron Collider (LHC). LHCb aims to search for possible contributions of New Physics to the loop correction of suppressed decays of beauty and charmed hadrons. B and c hadrons are abundantly produced in the proton-proton interactions of the LHC and the large rate offers the possibility for precision measurements, in addition to the possibility to measure or search for extremely rare decays. The advantage of $\mathrm{LHCb}$, in addition to the access to the expected $\approx 500 \mu \mathrm{b}$ production cross section of $\mathrm{B}$ hadrons at $14 \mathrm{TeV}$ centre of mass energy proton collisions, is the fact that all species of B-hadrons are produced, in particular $\mathrm{B}_{\mathrm{s}}$.

The currently installed and running detector is laid out as a single arm spectrometer between an inner square acceptance of $15 \mathrm{mrad}$ and an outer rectangular acceptance of $250 \times 300 \mathrm{mrad}$. The experiment is designed to trigger on and optimise the acceptance for $\mathrm{B}$ hadrons from the $b \bar{b}$ production, which is sharply peaked in the forward-backward direction. The possibility to tune the betratron amplitude $\beta^{*}$ at the $\mathrm{LHCb}$ interaction point allows the experiment can be run at lower instantaneous luminosity, and LHCb expects that after the LHC commissioning period, the $\mathrm{LHCb}$ design luminosity of $2 \times 10^{32} \mathrm{~cm}^{-2} \mathrm{~s}^{-1}$ will be comfortably reached. During the first running phase, when $\mathrm{LHCb}$ expects to accumulate $10 \mathrm{fb}^{-1}$ over a period of about 5 years, key measurements will include a first observation of the rare decay $B_{s} \rightarrow \mu^{+} \mu^{-}$, a measurement of the $\mathrm{CP}$ violating mixing phase in $B_{s} \rightarrow J / \psi \phi$ with an error at the level of the SM prediction, and the first precise measurements of the CKM angle $\gamma$ decay modes [2]. The upgrade will allow LHCb to operate with up to an order of magnitude increase in accumulated luminosity. Assuming that the LHC will reach luminosities of $\mathscr{O} 10^{33} \mathrm{~cm}^{-2} \mathrm{~s}^{-1}$ within the coming years, the upgrade of the LHCb detector is independent of the planned luminosity upgrade of the LHC but has to be coordinated with the machine schedule to allow the replacement of sub-detectors.

Precision measurements in the quark flavour sector are a sensitive probe for New Physics. Exciting hints for New Physics might be discovered already during the first phase of LHCb operation and might require more data to further establish any observed phenomena. Discoveries by the general purpose detectors ATLAS and CMS will also require higher precision of the flavor measurements to allow further exploration of any seen enhancements in the flavour sector. For these reasons, together with the fact that after the accumulation of the initial dataset the statistical improvements that can be expected while running at constant luminosity become too slow, an upgrade of the experiment is envisaged.

Running at significantly higher luminosities only makes sense if the current trigger rate limitation of $1 \mathrm{MHz}$ at which the detector is read out and which is imposed by the maximum bandwidth, can be overcome. The relevant trigger level (L0) is implemented in hardware and offers only little flexibility. If the luminosity is increased, the maximum trigger rate of $1 \mathrm{MHz}$ can be maintained in the current detector design only if the trigger cuts for the hadronic decay channels are tightened, leading to a severe drop in the trigger efficiency and very little overall gain. In addition to this, the parts of the detector which suffer from spill-over signal from the adjacent events will suffer a big increase in occupancy at high luminosity, and the events become correspondingly more complex. The current L0 decision is based only on a subset of detectors, and the time available for the decision is at most around $2.5 \mu \mathrm{s}$, which is inadequate to efficiently select B decays. It is therefore 
forseen that in the upgraded detector all sub-systems will be read out at an event rate of $40 \mathrm{MHz}$, and the data will be transferred to a computing farm running the trigger algorithms. This software trigger will be able to use all the event information, reconstruct all primary vertices, and combine impact parameter and transverse momentum information. The major implication of this decision is a change of the front-end electronics of the experiment. In addition, the detector will have to be improved to cope with up to an order of magnitude increase in the integrated radiation dose. The upgrade is planned in two phases: the first, major overhaul of the front end electronics to cope with a $40 \mathrm{MHz}$ readout rate, which also implies the complete replacement of certain sub-detectors, after which the experiment is expected to operate at about 5 times the design luminosity. A second phase of the upgrade, which would see an expansion of the CPU farm and further upgrades of certain parts of the detector, would allow a further doubling of the luminosity to $2 \times 10^{33} \mathrm{~cm}^{-2} \mathrm{~s}^{-1}$ and doubling of the hadron trigger efficiency.

\section{Physics opportunities at the LHCb upgrade}

As explained above, the upgrade will enable $\mathrm{LHCb}$ to pursue channels where the measurements are statistically limited at the current experiment, such as $\mathrm{B}_{\mathrm{s}} \rightarrow \phi \phi$, and to keep pace with precision theoretical predictions, which are expected to improve with time, and which allow tests for new phenomena in channels which are today limited by the theoretical understanding. An example is the indirect determination of the angle $\gamma$, which in 2010 is approximately $5^{\circ}$ and which by 2015 could shrink by a factor of around 5 thanks to significant improvements from lattice QCD [5], as illustrated in figure 1. Other physics opportunities after the upgrade are listed below: the given errors are estimated for an integrated luminosity of 10 and $100 \mathrm{fb}^{-1}$ expected before and after the upgrade, and a doubling of the hadron trigger efficiency after the upgrade.

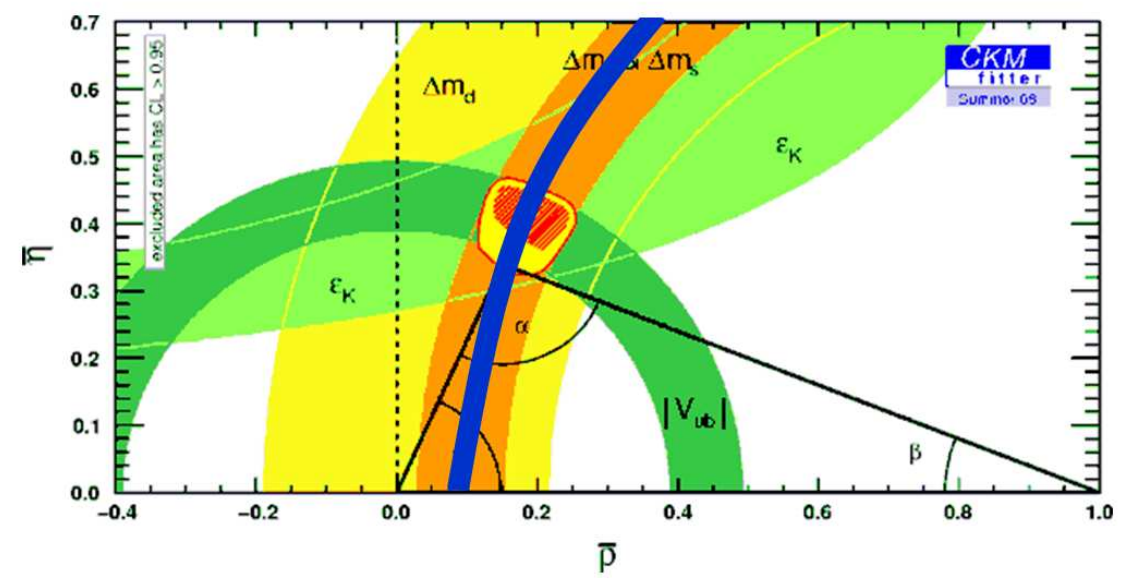

Figure 1: Constraints on the unitarity triangle, illustrating the indirect precision on the determination of $\gamma$, using currently available data. Superposed as the solid black curve, is the improvement expected with the anticipated improvements from lattice QCD calculations.

- Search for rare decays $\mathbf{B}_{\mathbf{s}} \rightarrow \mu \mu$ and $\mathbf{B}_{\mathbf{d}} \rightarrow \mu \mu$ The observation of the FCNC decays presents a very exciting prospect for $\mathrm{LHCb}$, which could provide either a clear indication of 
NP, or a strong constraint on NP physics models. The SM prediction has been calculated as $\mathscr{B}\left(\mathrm{B}_{\mathrm{s}} \rightarrow \mu \mu\right)=(3.35 \pm 0.32) \times 10^{-9}$ [7], giving a small, but relatively precise expectation. It is expected that LHCb can see a SM signal with an accumulated luminosity of $10 \mathrm{fb}^{-1}$, or sooner if there are significant enhancements due to NP. Certain models, such as MCPVMFV can not only give enhancements but can also predict a suppression of the branching ratio with respect to the SM value, depending on the relative value of the phases involved [9]. In addition, greater statistics than are achievable with the current LHCb detector are necessary in order to compete with the relative 10 percent precision of the SM prediction. Furthermore, the ratio of the branching fraction $\mathrm{B}_{\mathrm{d}} / \mathrm{B}_{\mathrm{s}} \rightarrow \mu \mu$ is tightly constrained and can also distinguish between SM and MFV models [8].

- Precise determination of $\phi_{s}$. The $\mathrm{B}_{\mathrm{s}}$ mixing phase $\phi_{s}$ is one of the most precisely predicted $\mathrm{CP}$ violating quantities in the $\mathrm{SM} ; \phi_{s}=-0.036 \pm 0.002 \mathrm{rad}[10]$. If there is a large NP effect in the $\mathrm{B}_{\mathrm{s}}$ sector, the current $\mathrm{LHCb}$ detector will certainly be able to observe first hints but a final clarification might need significantly more data. If it turns out to be small then the clean prediction must be matched by a precise measurement. The achieveable precision on this measurement with the current detector is expected to be around 0.01 in the golden $\mathrm{J} / \psi \phi$ channel alone [13], with enhancements expected from the addition of other channels such as $\mathrm{J} / \psi \mathrm{f}_{\mathrm{o}}(\pi \pi)$ [11]. The upgrade could enhance this precision to the level of 0.004 , approaching the SM precision. This measurement is of fundamental interest, leading for instance to a direct constraint on the unitarity triangle via its proportionality to $\eta$.

- $\mathbf{b} \rightarrow \mathbf{s}$ penguin decays. The penguin dominated decay $\mathrm{B}^{0} \rightarrow \phi \mathrm{K}_{\mathrm{s}}^{0}$ provides a measurement of $\sin \left(2 \beta^{\text {eff }}\right)$ which is very sensitive to NP. In the SM, the angle $\beta_{\text {eff }}$ is expected to be equal to that measured in $B_{\mathrm{d}} \rightarrow J / \psi K_{\mathrm{S}}$ decays with small theoretical uncertainty. NP in the $b \rightarrow s$ transition may lead to additional CP violating phases, and a deviation from the SM prediction. Improved measurements are needed to shed light in this area. At the upgrade we hope to collect up to 70000 events, leading to a precision of 0.025 , extrapolating from [12]. This channel can be complemented by the $\mathrm{B}_{\mathrm{s}}^{0} \rightarrow \phi \phi$ decay, in which the penguin and box contributions to the mixing phase are expected to cancel, and where NP contributions could affect this cancellation and result in a measureable $\mathrm{CP}$ asymmetry. This measurement is unique to $\mathrm{LHCb}$. After the upgrade up to $0.5 \mathrm{M}$ events are expected, leading to a precision of 0.02 , extrapolating from [13].

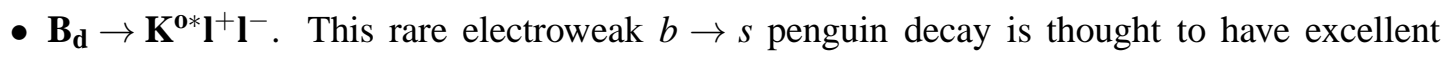
sensitivity to physics beyond the SM. It provides a host of sensitive observables, such as the forward backward asymmetry measured for the lepton with respect to the B flight direction in the dilepton rest frame. The dependence of the asymmetry on the dilepton invariant mass can distinguish between different NP physics models. In particular the zero crossing point of the asymmetry has very small theoretical uncertainties. The position of the zero crossing point will be measured already very precisely with the current LHCb detector. However a full angular analysis which provides the best discriminating power is only possible with an accumulated luminosity of greater than $10 \mathrm{fb}^{-1}$. After the upgrade the yield is expected to be of the order of 0.35 million events [14], giving access to further observables such as the 
transverse asymmetries, which are sensitive to NP and constrain SUSY models which are currently compatible with the available data [16].

Other measurements with the upgraded detector not discussed above include an anticipated sub-degree precision on the tree level $\gamma$ measurements, improvements of up to a factor 5 in the measurement of $\gamma$ from charmless hadronic B decays, the search for RH currents in radiative $B_{s}$ decays, and opportunities in charm physics.

\section{LHCb detector upgrade}

As explained in the introduction above, in order to overcome the current $1 \mathrm{MHz}$ bottle neck in the system, the LHCb upgrade relies on a complete change of trigger strategy, and a readout of the entire detector at $40 \mathrm{MHz}$. The trigger, which will be fully software based, will operate on a CPU farm with an input event rate $30 \mathrm{MHz}^{1}$. The goal is to double the trigger efficiency for hadronic channels, and make the trigger scalable with luminosity. As all the event information will be available, the trigger is able to reconstruct primary vertices, and combine cuts on the transverse momentum and impact parameters. A key point of the upgraded trigger strategy is the fact that the inbuilt flexibility will allow the experiment to adapt to the changing physics landscape over the next decade. The first phase of the upgrade is envisaged to be installed around 2015-16, in synchronisation with LHC machine shut downs.

In order to cope with the huge increase in event rate there will be substantial modifications to the readout scheme and DAQ system. The entire event selection will be performed in a large EFF (Event Filter Farm), where the trigger algorithm is executed on complete events. A "ratecontrol trigger" is envisaged to cope with the fact that the DAQ system will be staged, which will enrich the sample in desired events, using information available from a subset of detectors, similarly to the current $\mathrm{LHCb} \mathrm{L} 0$ trigger. The data rate from the detector is expected to correspond to approximately 110003.2 Gigabit links, which will be sent to a newly designed readout board with at least $40 \mathrm{Gbit} / \mathrm{s}$ output bandwidth and the possibility to process $400 \mathrm{Gbit} / \mathrm{s}$ of input data. Another integral part of the upgraded DAQ system is the use of the Gigabit Bidirectional Trigger and Data link (GBT). For more details see [2, 3].

The design of the current LHCb detector has been described in [1]. The experimental environment at the upgrade poses additional challenges to the experiment. There will be up to a tenfold increase in integrated radiation dose. The current detector was designed to withstand an accumulated dose equivalent to $20 \mathrm{fb}^{-12}$, and the increase in radiation beyond these levels will effect mainly the areas at large pseudorapidity, such as the trackers and the inner part of the calorimeter. Clearly, some running experience with the current detector at the LHC will be necessary to understand the exact evolution of the detector performance. The VELO, which is expected to deteriorate at lower integrated luminosities will in any case be completely replaced. Another challenge will be the detector occupancies and the tracking performance at higher luminosities. The occupancy increase is due not only to the increased pile-up, but also due to the fact that more bunches are filled, and the detector as a whole will suffer more from spillover from the previous and next bunch

\footnotetext{
${ }^{1}$ corresponding to the non-empty bunch crossing rate at $\mathrm{LHCb}$

${ }^{2}$ with the exception of the silicon detector, which is forseen to be replaced earlier
} 
crossings. In the case of the silicon detectors this spillover can be dealt with thanks to the fact that the full information from the adjacent events is available on the readout boards. Taking as a benchmark $\mathrm{B}_{\mathrm{s}} \rightarrow \phi \phi$ events, the occupancy in the VELO and Silicon Tracker is expected to increase by a factor of about 2.4 for an order of magnitude increase in luminosity. The impact of the increased luminosity on the tracking efficiency has been studied up to a luminosity of $11 \times 10^{32} \mathrm{~cm}^{-2} \mathrm{~s}^{-1}$. The total yield of tracks, folding together the increased production with the tracking efficiency drop for both two and four prong decays is shown in figure 2, and shows linear behaviour. However, in a possible second phase of the upgrade, reaching higher luminosity, the occupancies in the straw tubes of the Outer Tracker reach unacceptable levels. Possible solutions include increasing the area coverage of the Inner Tracker, and using faster gas compositions for the straw tubes. Material budget is also a critical issue for the upgrade, as any increase in material will exacerbate the occupancy effects. The drive to keep material at a minimum is equal to, if not greater than for the current detector. In the following sections the upgrade strategy is discussed for the various subdetectors.

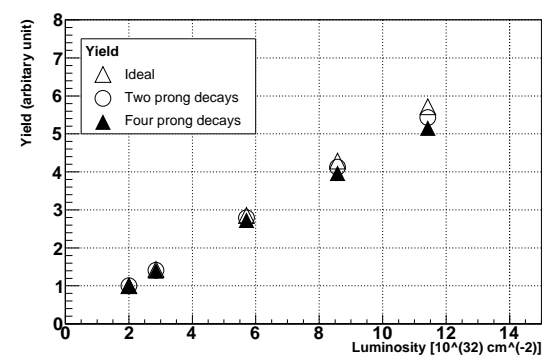

Figure 2: The figure displays the single track reconstruction efficiency, folded together with the increased event yield, to give the expected evolution of yield as a function of luminosity, evaluated with the full $\mathrm{LHCb}$ simulation software, for two and four prong decays. The yield is normalised to the number of tracks at a luminosity of $2 \times 10^{32}$.

\subsection{Vertexing: The VELO}

The current VELO is manufactured with $84 R$ and $\phi$ measuring silicon strip sensors operated in a secondary vacuum inside the LHC beampipe. The detector is divided into two moveable halves, allowing it to retract during LHC injection, and separated from the primary vacuum with a $300 \mu \mathrm{m}$ thin aluminium foil, which is corrugated in such a manner as to reduce as far as possible the material traversed by particles before they reach the silicon sensors. For a full description see [4] and references therein. The current VELO cannot be operated to the expected radiation levels of $0.8 \times 10^{16} \mathrm{n}_{\mathrm{eq}} \mathrm{cm}^{-2}$, corresponding to a total ionising dose of about $370 \mathrm{MRad}$, and it is mandatory to redesign the modules to withstand thermal runaway issues after high irradiation, as well as completely replacing the front end electronics to be compatible with the $40 \mathrm{MHz}$ readout.

The current baseline design is for a pixel based device with a $200 \mu \mathrm{m}$ thick diamond cooling spine which extends to the tip of the silicon. The cooling is envisaged to be a mixed phase $\mathrm{CO}_{2}$ system similar to the current VELO, with the cooling capillaries at approximately $-40^{\circ}$ running around the edge of the silicon as far as possible out of the acceptance. Each module consists of two 6 chip ladders, with the chips mounted on opposite side of the diamond cooling plane to 
facilitate the readout. The Timepix [17] chip, from the Medipix family of chips, has been identified as an excellent candidate for the FE electronics solution. The current chip contains $256 \times 256$ pixels of $55 \mu \mathrm{m}$ by $55 \mu \mathrm{m}$ square and the overall dimensions of the chip are $14 \mathrm{~mm} \times 14 \mathrm{~mm}$. Each pixel digitizes the charge by measuring time over threshold (TOT) and provides at least 4 bit equivalent precision over a range of input charges up to $50 \mathrm{ke}^{-}$. The chip is 3 side buttable with on one side a dead region containing the peripheral circuitry and bonding pads (with an option for the use of through-silicon-via technology). The chip is currently built in $0.25 \mu \mathrm{m}$ technology, and will move to $130 \mathrm{~nm}$ technology in the next design cycle, corresponding to what has already been implemented for the Medipix 3 [18] chip. New irradiation results from this chip show that integrated doses of $500 \mathrm{MRad}$ are within reach [19], which is in the range of what is required for the VELO. In a testbeam in 2009 the first demonstration of the use of Timepix bonded sensors for charged particle tracking showed excellent performance [20]. The chip would need modifications to be suitable for the $40 \mathrm{MHz}$ sustained readout rate at LHCb. It is anticipated that the occupancies at the highest luminosity would reach 6 hits per chip per beam crossing, for the most occupied chips. With a mean cluster size of up to 3 (depending on detector thickness), and the anticipated overheads of 12 bits for the time stamping, 4 bits for the TOT information and a 16 bit address, this leads to readout bandwidth of up to $23 \mathrm{Gbit} / \mathrm{s}$ per chip. This issue is being addressed with a new digital readout architecture, which uses $4 \times 4$ super pixel arrays to share logic and provide on-pixel clustering.

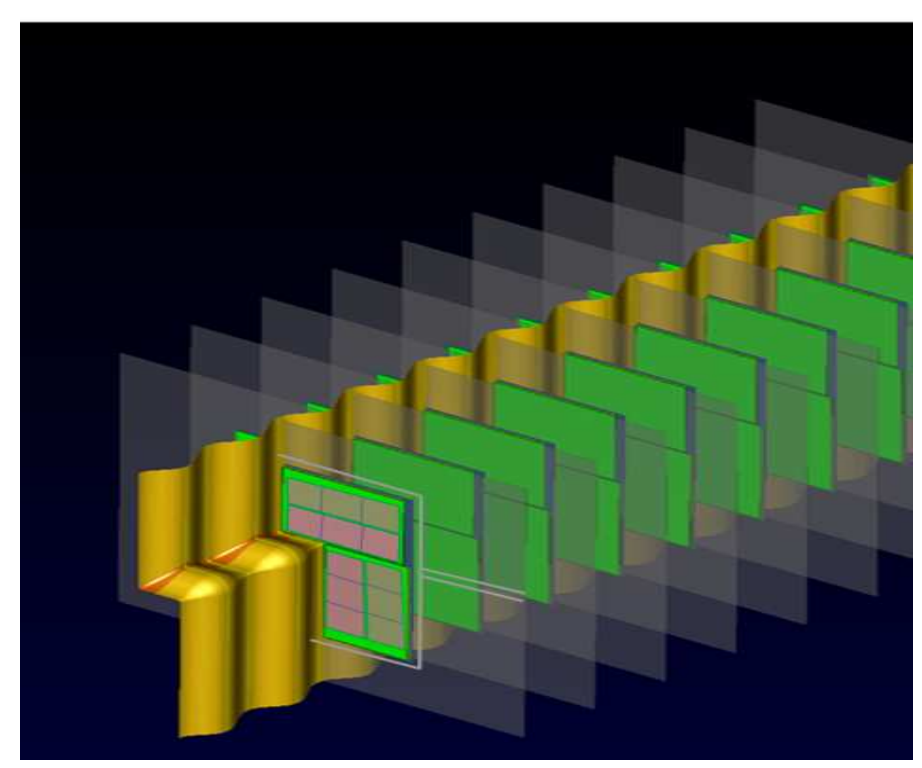

Figure 3: Sketch showing the possible arrangement of pixel modules around an L-shaped RF foil. On the first module the $2 \times 6$ chip arrangement is illustrated. Each row of three chips is on an opposite side of the module. The benefit of this arrangement is that it enables the chip to more easily cope with the very high and non-uniform occupancy: the readout of a pixel column always samples both a high and low occupancy region of the chip.

Another crucial component of the upgrade project is the design of the foil separating the primary and secondary vacuum. The foil used in the current VELO dominates the material seen before 
the first measured point, and in order to optimise the impact parameter resolution it is desirable to reduce this amount of material for the upgrade. It is also not clear that the hot pressing technique used to manufacture the current foil can be used for the new pixel geometry. For this reason, a new technique is under investigation with the company CMA ${ }^{3}$ which uses a carbon fiber composite substrate, with the goal of manufacturing an object with the required mechanical tolerances, resistance to radiation and small differential pressures, and an overall reduction in the amount of material.

\subsection{Tracking Systems}

Operating at high luminosity will produce high occupancy and ghosts in the tracking system, particularly in the Outer Tracker, where a drift-chamber technology is used and where, because of the drift-time, the charge collection time takes between 50 and $75 \mathrm{~ns}$. The current layout of the tracking stations is shown in figure 4 a), where the outer tracker straw part is seen (in yellow) surrounding the innermost tracking boxes which use ladders of silicon strip sensors. The silicon ladders themselves, which are integrated to the current $1 \mathrm{MHz}$ readout electronics have to be completely replaced (with the possible option of removing and rebonding the silicon). In a possible second phase of the upgrade the area coverage of the Inner Tracker would be increased in order to reduce occupancy in the Outer Tracker. In addition the full silicon tracker station (TT) in front of the LHCb magnet has to be completely replaced. The material budget, which has an impact on the momentum resolution and pattern recognition performance, will be a critical issue, and radiation resistance must also be taken into account. An alternative solution is to replace the entire tracking station with a fiber tracker with mixed fiber dimensions, as illustrated in figure $4 \mathrm{~b}$ ), ( $250 \mathrm{~mm}$ for the inner part, 700-1000 mm for the outer part) which could be read out with Silicon Photo Multiplier detectors or conventional MaPMT. The advantages of such a solution would be a simplified configuration of services (no cables, cooling or frame within the acceptance) along with a flexible geometry, which could bring the modules closer to the beam pipe. The signal light is transported to the SiPM readout and hybrids located outside the acceptance, simplifying the design and the electronics development due to the lower radiation. The spatial resolution has been demonstrated in a testbeam to be adequate [21]. The problems to be addressed include the development of new electronics for the SiPM readout, and studies of the fiber to fiber coupling, for readout outside the acceptance.

\subsection{Particle ID: the RICH systems}

The LHCb experiment currently uses a system of two independent RICH detectors; an upstream "RICH1", which uses Aerogel and $\mathrm{C}_{4} \mathrm{~F}_{10}$ radiators, and a downstream "RICH2", with a $\mathrm{CF}_{4}$ gas radiator. The combined information from these devices covers the momentum range from $1 \mathrm{GeV} / \mathrm{c}$ up to about $100 \mathrm{GeV} / \mathrm{c}$, with the aerogel dedicated to the region between 1 and $10 \mathrm{GeV} / \mathrm{c}$. The photon detectors used are customised HPDs (Hybrid Photon Detectors) with the readout electronics embedded within the vacuum photo-tube. For this reason, these detectors must be completely replaced for the upgrade, in order to enable the required $40 \mathrm{MHz}$ data transmission. The

\footnotetext{
${ }^{3}$ Composite Mirror Applications, Tucson, AZ
} 


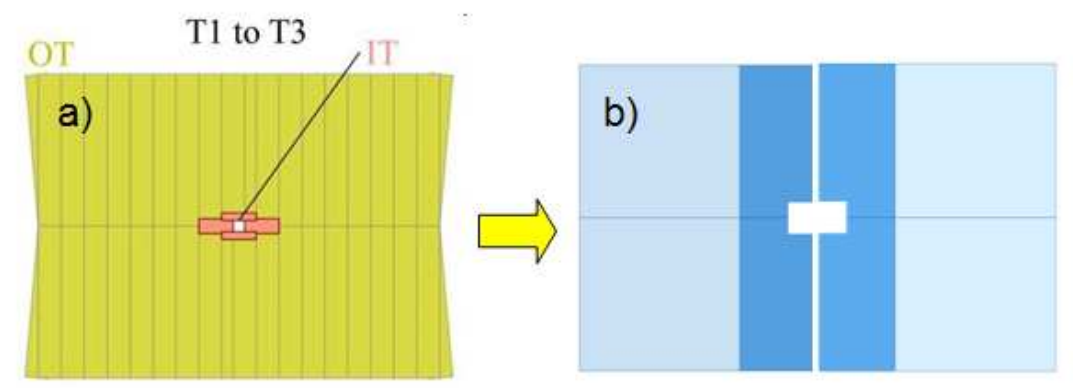

Figure 4: Sketch illustrating a) the current layout of the three tracking stations T1 to T3 of LHCb, with the central silicon detector boxes(labelled IT) surrounded by the large straw chambers (labelled OT), and b) the possible upgraded layout to a fiber tracker solution, with two regions of different fiber dimensions.

RICH R\&D programme is investigating alternative photon detectors for the upgraded RICH. Driving issues are the geometrical coverage, the performance of the detectors in the fringe field region where they must operate, the cross talk and the spillover. A promising candidate appears to be the Hamamatsu manufactured MaPMT. A dedicated electronics chip compatible with the overall 40 $\mathrm{MHz}$ readout architecture must also be developed. Apart from this major change, the RICH geometry could be maintained in the current configuration, or alternative solutions could be envisaged. The current aerogel implementation has a low photon yield, and its use will become challenging with the higher occupancy of the upgrade. For this reason one of the most promising configurations seems at present to remove the aerogel, giving the additional advantage of decreasing material in the tracking volume, and provide the low momentum coverage with a new Time Of Flight system which could be located, for example, after RICH2. This new concept, known as the "TORCH" [22] would be based on the installation of a $7.4 \mathrm{~m} \times 6.1 \mathrm{~m}$ quartz plate located $12 \mathrm{~m}$ from the interaction point, illustrated in figure 5. Photons produced by traversing particles travel by total internal reflection to the edges of the plate, where standoff boxes located outside the spectrometer acceptance focus them onto the photodetector planes. Combining together the spatial measurements of the photons with their time of arrival allows the time of flight of the particle to be reconstructed. A precision of $70 \mathrm{ps}$ for the individual photons (10-20 ps for the charged particles) should be adequate for the PID requirements. Detailed simulations are currently underway to establish a reliable pattern recognition strategy and to investigate possible solutions with the TORCH placed closer to the interaction point, which would save on the volume of quartz required. A promising photon detector for the TORCH readout could be the Micro Channel Plate, which is an intrinsically fast, operating around 50-100 ps and which, due to its small size has a high magnetic field tolerance [23]. Until recently, the lifetime of MCPs was an issue for the levels of integrated ionisation dose expected at $\mathrm{LHCb}$; this has been recently cured at Hamamatsu by the addition of a thin foil between the two MCP plates which prevents ion feedback [24].

\subsection{Calorimetry}

The current calorimeter system of LHCb is already configured to provide trigger signals at 40 


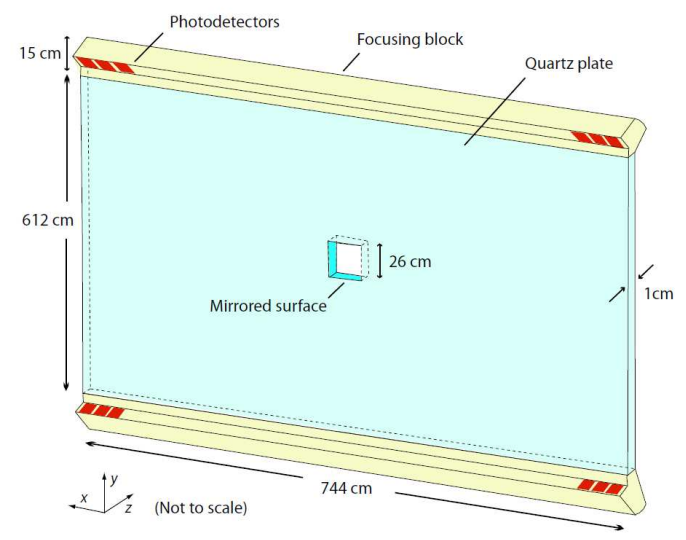

Figure 5: Schematic layout of the TORCH detector. Focusing elements have only been shown attached to the upper and lower edges, for clarity. The photodetectors extend along the full length of each edge; only a few are illustrated here.

$\mathrm{MHz}$, and the modifications needed for the front end electronics are less drastic than in the silicon and RICH subsystems, involving mainly the upgrade of the front end boards and the reduction of the gain of the photo multipliers. The radiation tolerance of the calorimeters is currently an open issue, and studies are ongoing to measure the performance of the current detector given the expected dose together with the annealing performance. The part of the calorimeter mainly affected by this issue is the inner part of the ECAL, which is expected to accumulate around 1.25 MRad per upgrade year during the first phase. The current limit to which tests have been performed is 2.5 MRad, and an R\&D programme is underway to collect more comprehensive data. In September 2009 calorimeter modules were placed into the tunnel upstream of $\mathrm{LHCb}$, which is an area where a high dose can be quickly accumulated. If current estimates are confirmed, the innermost part of the ECAL would have to be replaced after about 6 years of running, when the constant term in the resolution function will have almost doubled. Another important issue for the calorimeter upgrade running is the impact of pile-up, which affects the energy resolution performance, and can impact in particular the low $\mathrm{p}_{\mathrm{T}}$ and $\gamma$ physics. However the first $\mathrm{LHCb}$ results show a better than expected resolution in the current calorimeter and this effect will be evaluated in the light of the current data taking.

\subsection{Muon System}

As for the calorimeters, the muon system is already read out at $40 \mathrm{MHz}$ and yes/no information is sent to the level zero trigger. The system reconstructs hits via crossed xy pads, and therefore an impact of the higher luminosity could be to create an unacceptable level of ghost hits. Apart from this there are two main concerns; the dead time due to the high rate in the inner regions, and aging due to radiation in the inner regions. To understand the full impact of the detector resistance (channel rates and doses) and the granularity issue a careful evaluation with the first LHC data is needed. It is anticipated that rates of $1 \mathrm{MHz}$ per channel might be exceeded for the innermost portions of the detector at the highest luminosity, which would affect the efficiency. In this case 
a replacement could be envisaged, with smaller pad readout or possibly with large area GEMs or MWPCs.

\section{Conclusions}

The LHCb experiment plans to install a fully upgraded detector during a long shutdown around 2015-2016. The silicon detectors and photomultipliers will be completely replaced, and the front end electronics will be modified to allow a full readout of the detector at $40 \mathrm{MHz}$. The trigger performance of the upgraded detector is expected to double in efficiency for hadronic channels, compared to the current detector. In addition the yield for hadronic channels will scale with luminosity for both hadronic and leptonic channels. In a first phase the upgraded detector will run at a luminosity of $10^{33} \mathrm{~cm}^{-2}$. The recorded data will significantly enhance the capability of the experiment to perform precision measurements and NP searches in the flavour sector.

\section{References}

[1] “The LHCb Detector at the LHC”, 2008 JINST 3 S08005

[2] CERN-LHCC-2008-007

[3] P. Moreira, T. Toifl, A. Kluge, G. Cervelli, F. Faccio, A. Marchioro, J. Christiansen, Nucl. Sci. Symp. Conf. Record, 2 (2000) pp. 96-99

[4] M.G. van Beuzekom, Nucl. Instrum. Meth., 21 October 2008, Pages 21-24, ISSN 0168-9002.

[5] E. Gamiz et al., "Neutral B Meson Mixing in Unquenched Lattice QCD”, arXiv:0902.1815

[6] Modified from http://ckmfitter.in2p3.fr/plots_Moriond09/

[7] M. Blanke, A.J. Buras, D. Guadagnoli and C. Tarantino, arXiv:hep-ph/0604057 (2006)

[8] Buras: hep-ph/060405

[9] J. Ellis et al., Phys Rev D76:115011, 2007

[10] A. Lenz and U. Nierste, J. High Energy Physics 06, 072 (2007) [arXiV:hep-ph/0612167]

[11] S. Stone, L. Zhang, LHCb-PUB-2009-015

[12] Y. Xie, LHCb-2007-130

[13] O. Leroy, Proceedings, this conference.

[14] W. Reece, Proceedings, this conference.

[15] htp://www.slac.stanford.edu/xorg/hfag/triangle/moriond2009/plots/sPengS_CP.png

[16] U. Egede, et al. JHEP11 (2008) 32

[17] X. Llopart et. al., "Timepix, a 65k programmable pixel readout chip for arrival time, energy and/or photon counting measurements", NIM A 581 (2007) 485-494.

[18] R. Ballabriga et al., IEEE Trans. Nucl. Sci., Vol. 54, No. 5, October 2007.

[19] R. Plackett, CERN-2009-006, Pages 157-600, ISBN 978-92-9083-335-2

[20] P. Collins et al. To appear in Nuclear Inst. and Methods in Physics Research, A, 10.1016/j.nima.2010.04.108 
[21] R. Greim, H. Gast, T. Kirn, T. Nakada, G. Roper Yearwood, and S. Schael, Proceedings of he 31st ICRC, LODZ 2009

[22] R. Forty, M. Charles, CERN-LHCb-PUB-2009-030

[23] NIM A 567 (2006) 124-128

[24] T. Iijima, To appear in proceedings of RICH 2010 